\title{
Hirsutane-Type Sesquiterpenes with Uncommon Modifications from Three Basidiomycetes
}

Johannes C. Liermann, ${ }^{\dagger}$ Anja Schüffler, ${ }^{\ddagger}$ Beate Wollinsky, ${ }^{\ddagger}$ Judith Birnbacher, ${ }^{\ddagger}$ Heinz Kolshorn, ${ }^{\S}$ Timm Anke, ${ }^{\perp}, *$ Till Opatz, ${ }^{\dagger}, *$

${ }^{\dagger}$ Institut für Organische Chemie, Universität Hamburg, Martin-Luther-King-Platz 6, D-20146 Hamburg, Germany, Lehrbereich Biotechnologie, ${ }^{\ddagger}$ Technische Universität Kaiserslautern, PaulEhrlich-Str. 23, D-67663 Kaiserslautern, Germany, ${ }^{\S}$ Institut für Organische Chemie, Universität Mainz, Duesbergweg 10-14, D-55128 Mainz, Germany, and ${ }^{\perp}$ Institut für Biotechnologie und Wirkstoff-Forschung e. V., Erwin-Schroedinger-Str. 56, D-67663 Kaiserslautern, Germany

E-Mail: opatz@chemie.uni-hamburg.de

\section{Supporting Information}

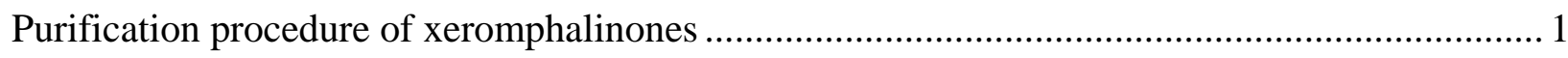

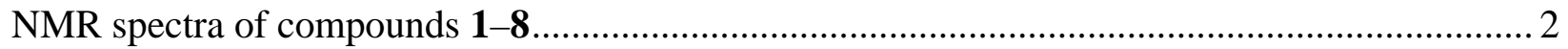




\section{Scheme 1. Purification procedure of xeromphalinones}

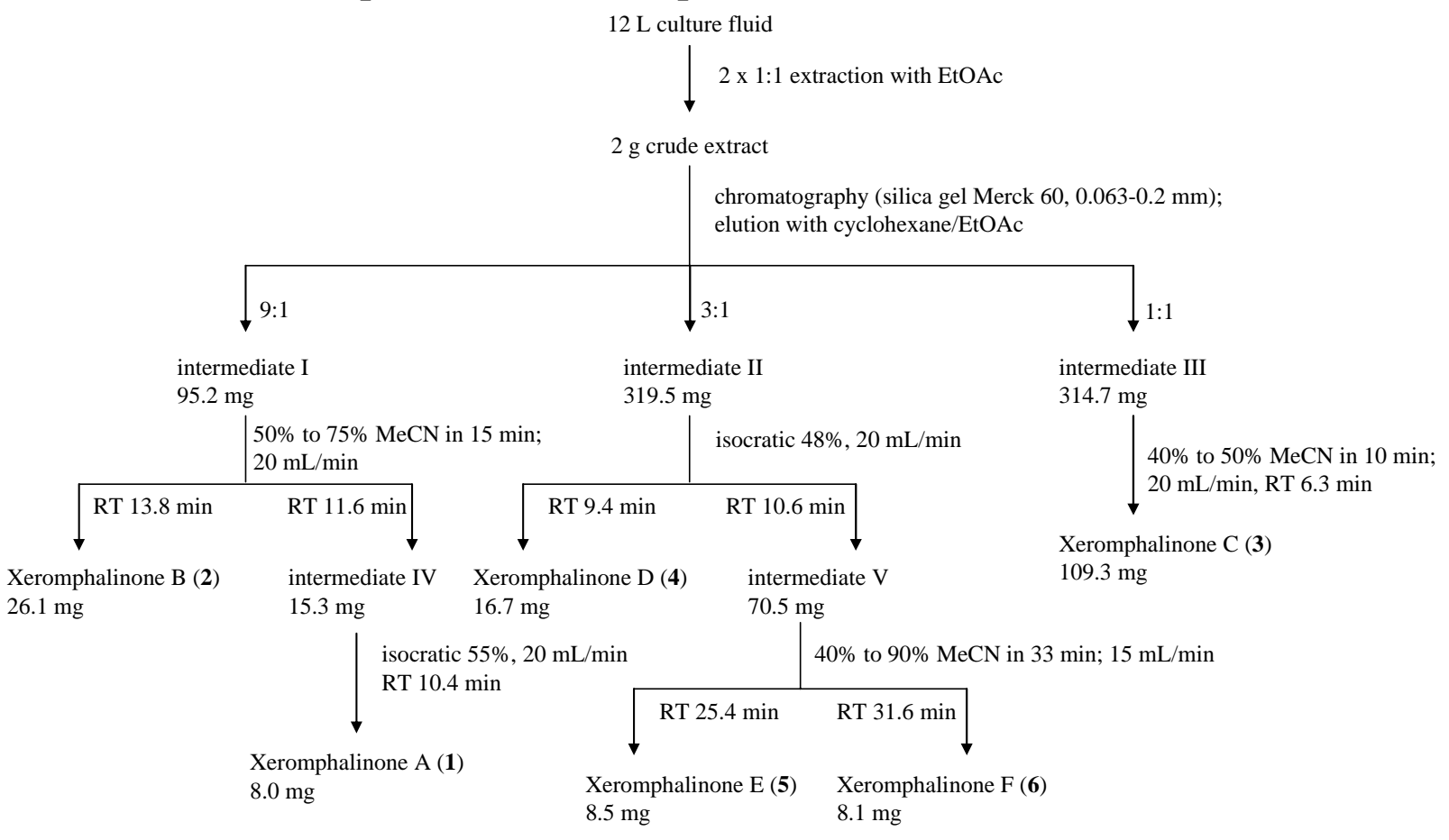


${ }^{1} \mathrm{H}$ NMR (400 MHz, $\left.\mathrm{CDCl}_{3}\right)$ of xeromphalinone A (1)

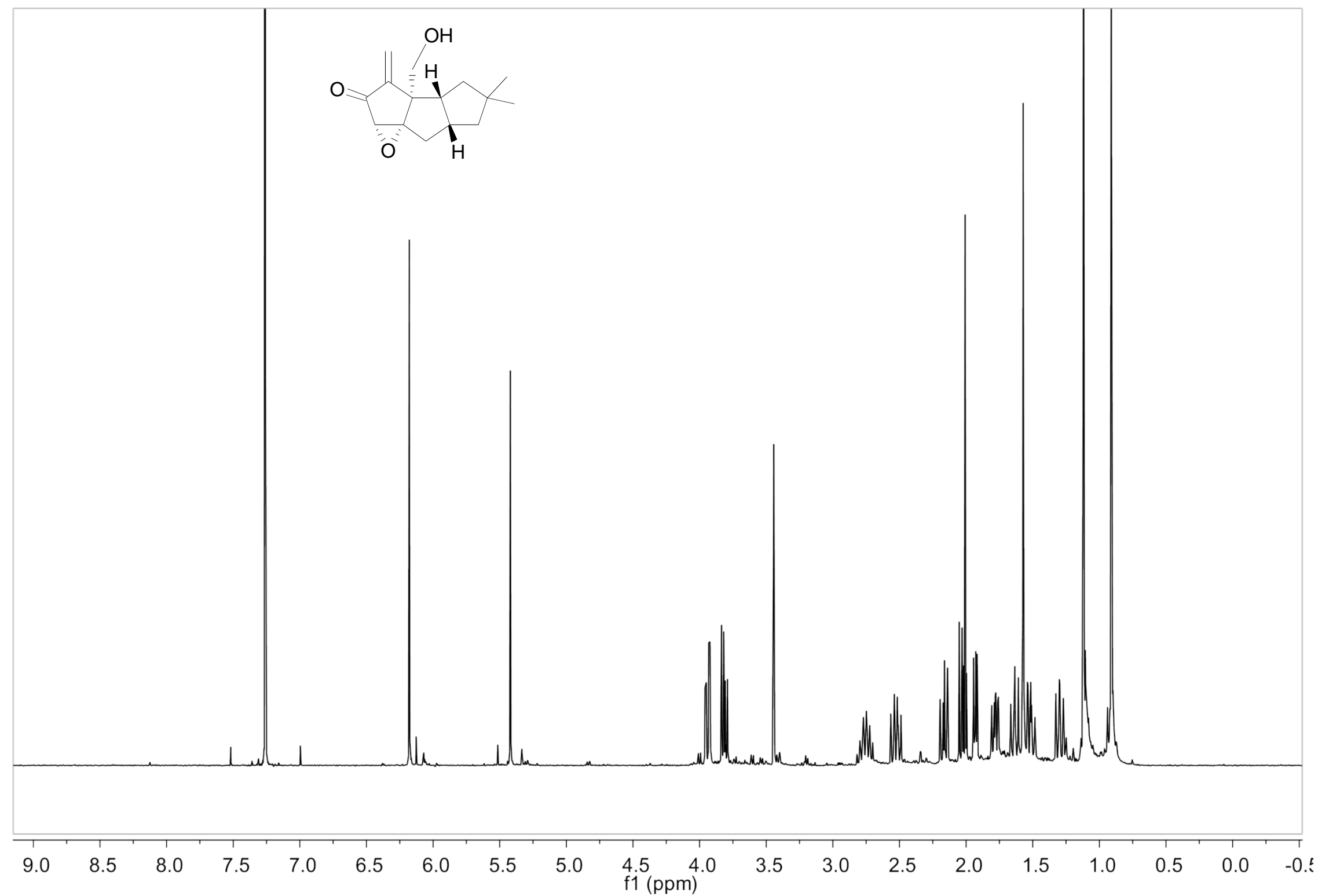


${ }^{13} \mathrm{C}$ NMR (101 MHz, $\left.\mathrm{CDCl}_{3}\right)$ of xeromphalinone A (1)

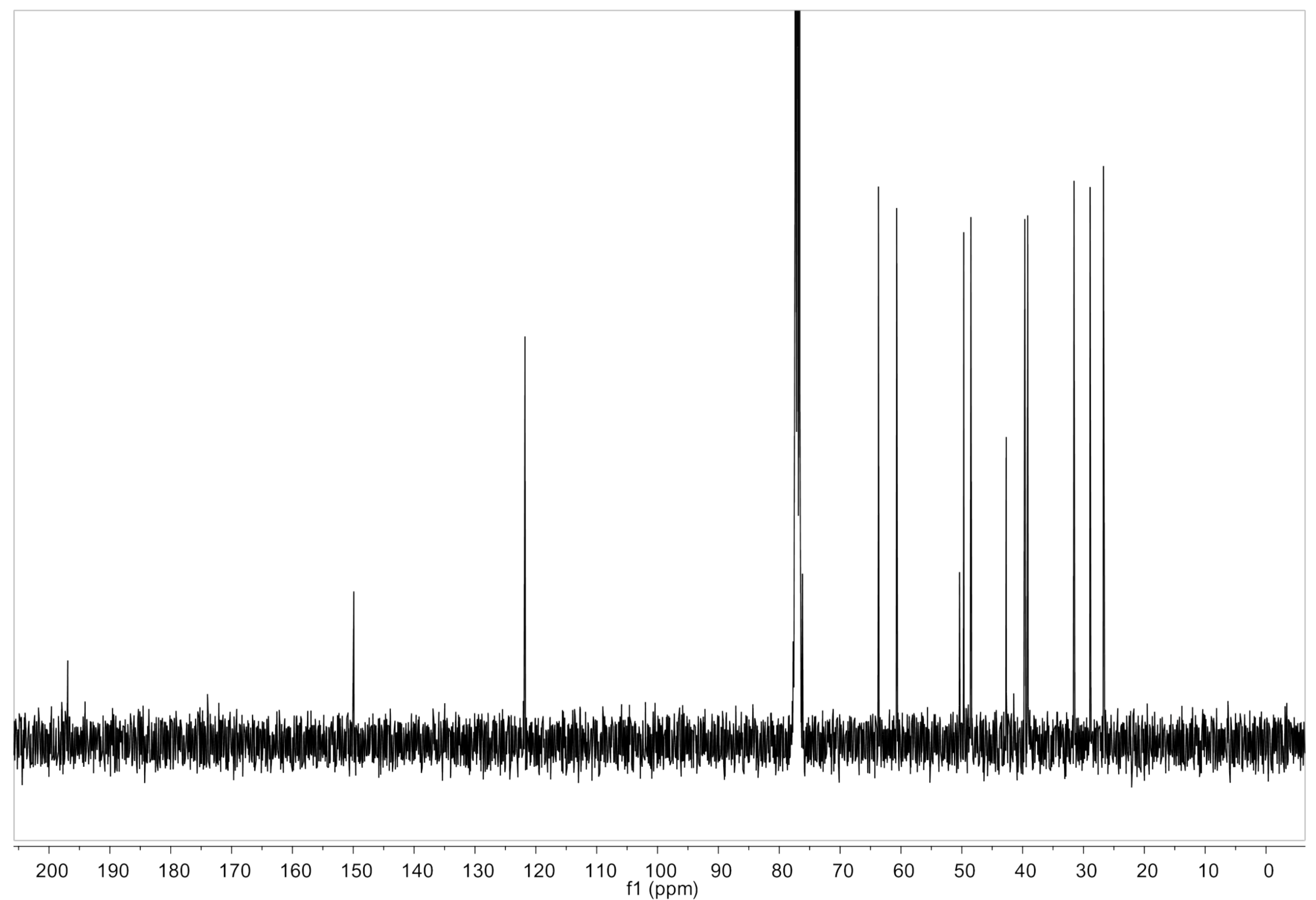


${ }^{1} \mathrm{H}$ NMR (400 MHz, $\mathrm{CDCl}_{3}$ ) of xeromphalinone B (2)

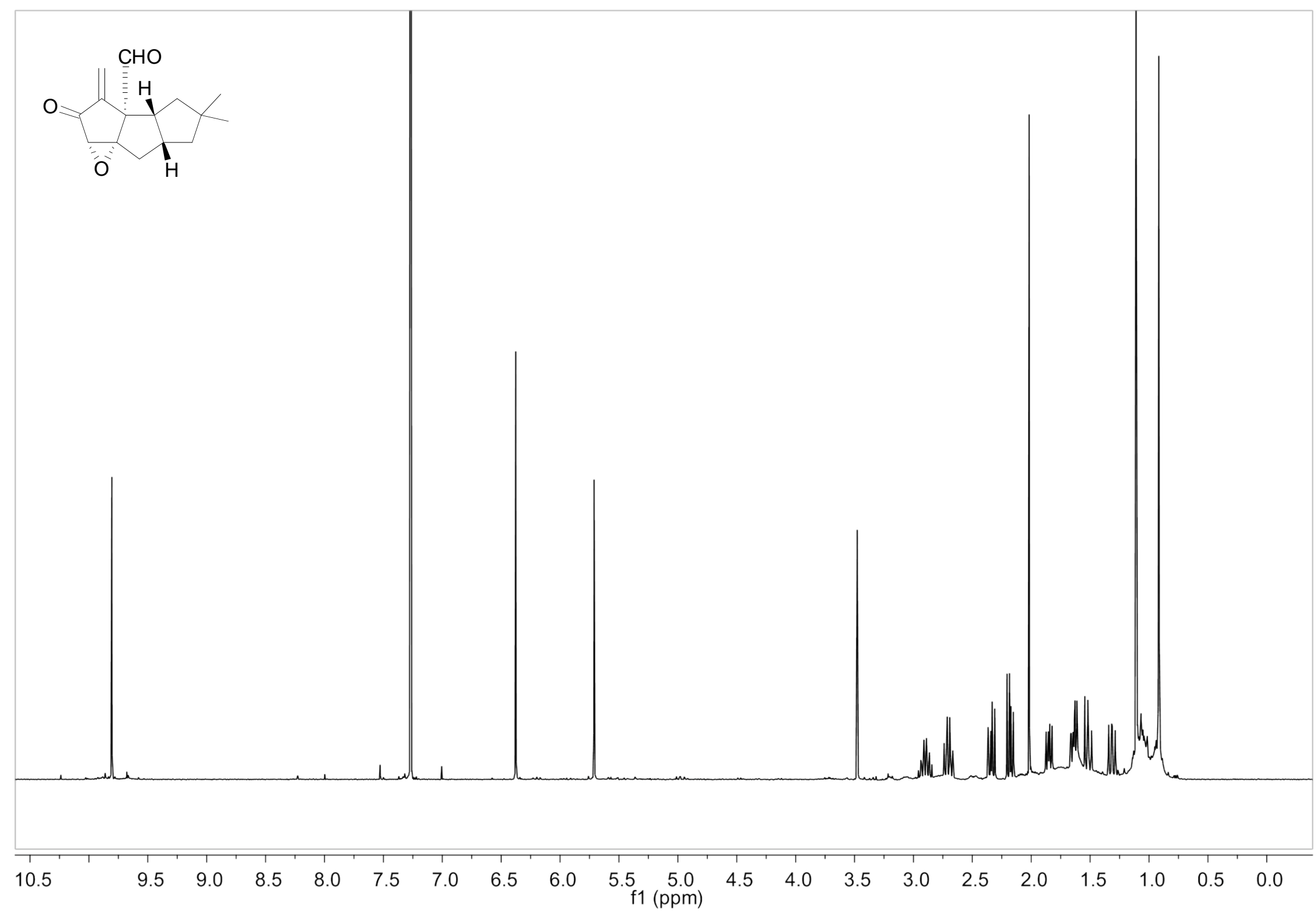


${ }^{13} \mathrm{C}$ NMR (101 MHz, $\mathrm{CDCl}_{3}$ ) of xeromphalinone $\mathrm{B}$ (2)

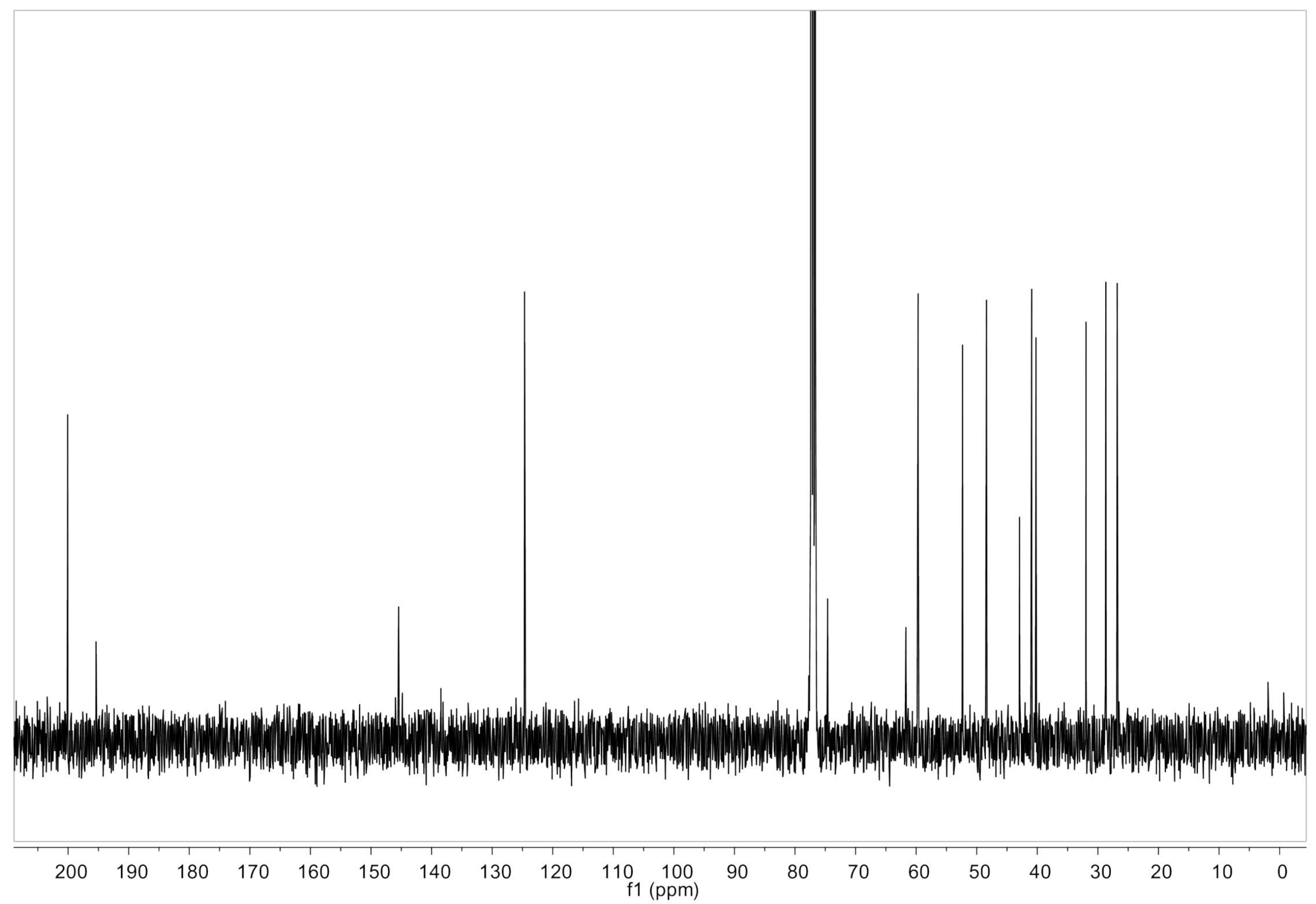


${ }^{1} \mathrm{H}$ NMR (400 MHz, MeCN- $d_{3}$ ) of xeromphalinone C (3)

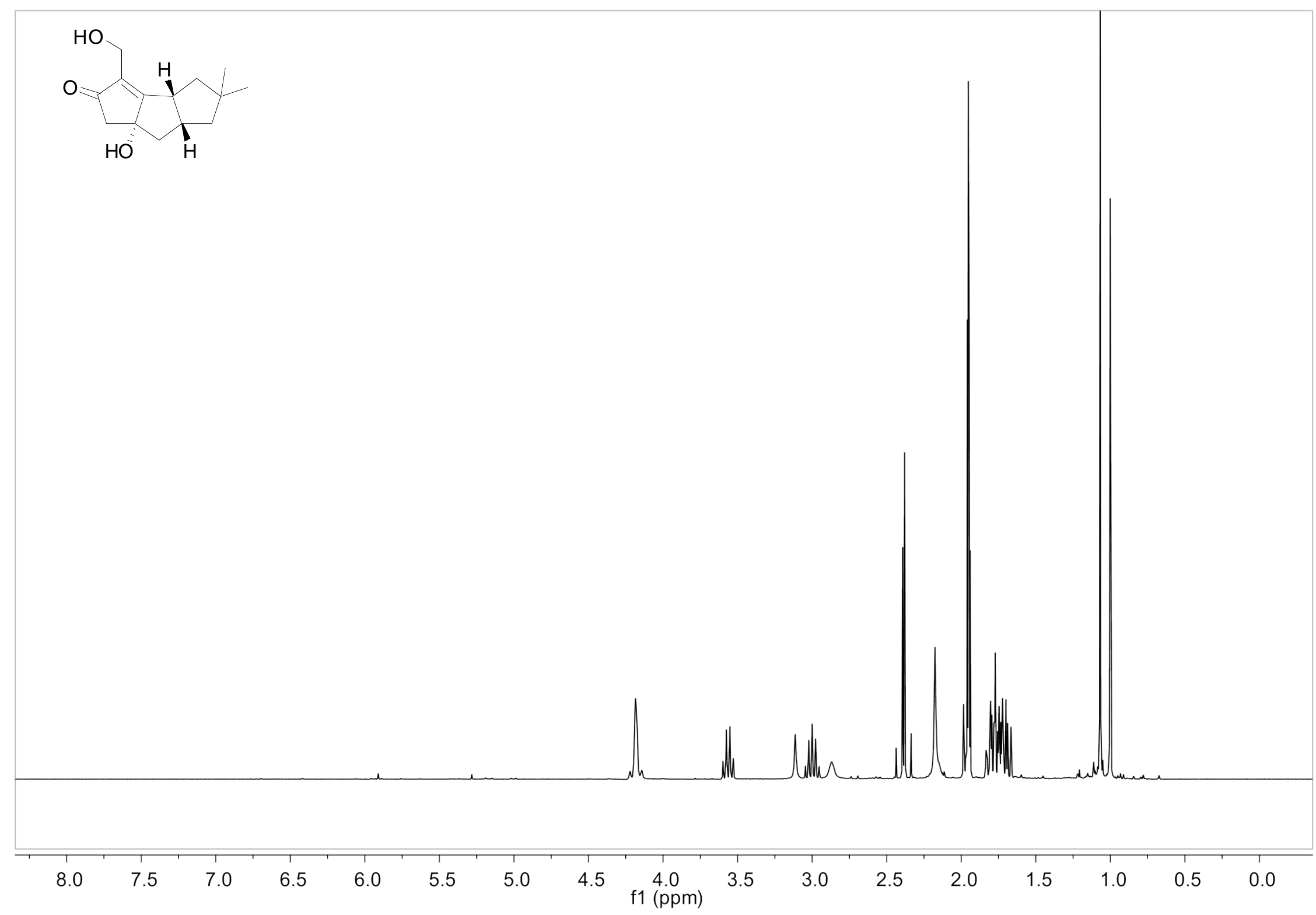


${ }^{13} \mathrm{C}$ NMR (101 MHz, MeCN- $d_{3}$ ) of xeromphalinone C (3)

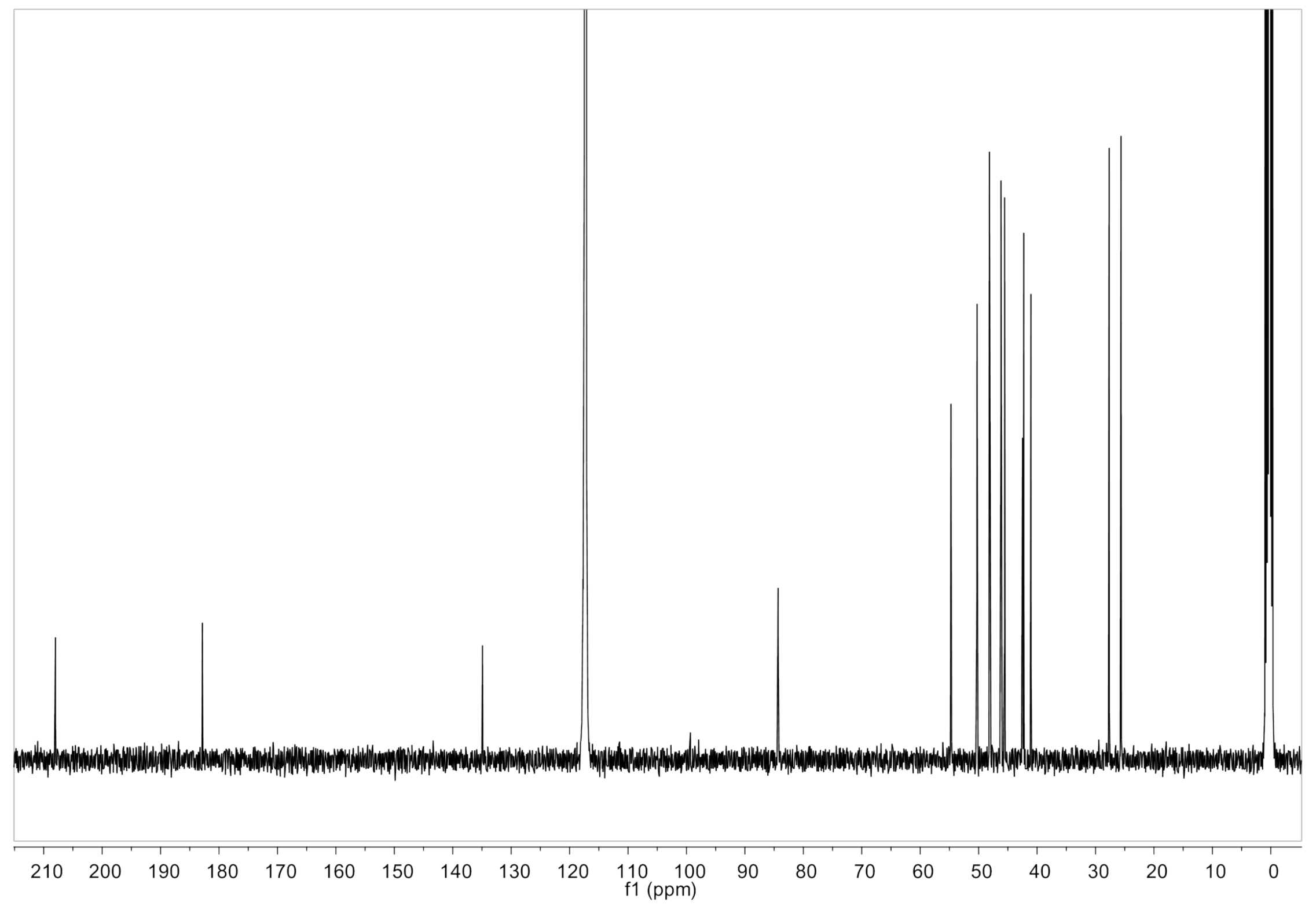


${ }^{1} \mathrm{H}$ NMR (400 MHz, $\mathrm{CDCl}_{3}$ ) of xeromphalinone D (4)

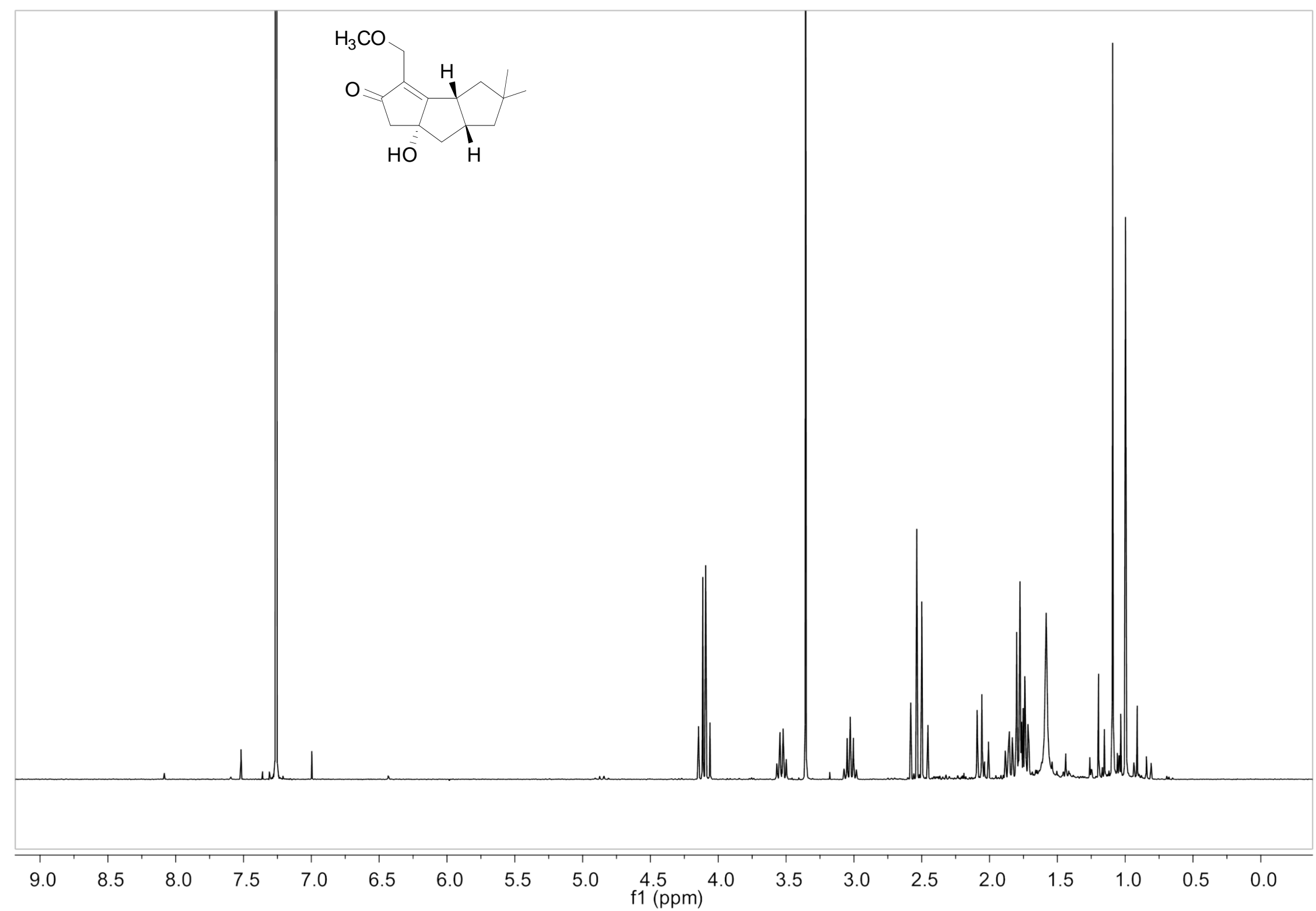


${ }^{13} \mathrm{C}$ NMR (101 MHz, $\left.\mathrm{CDCl}_{3}\right)$ of xeromphalinone D (4)

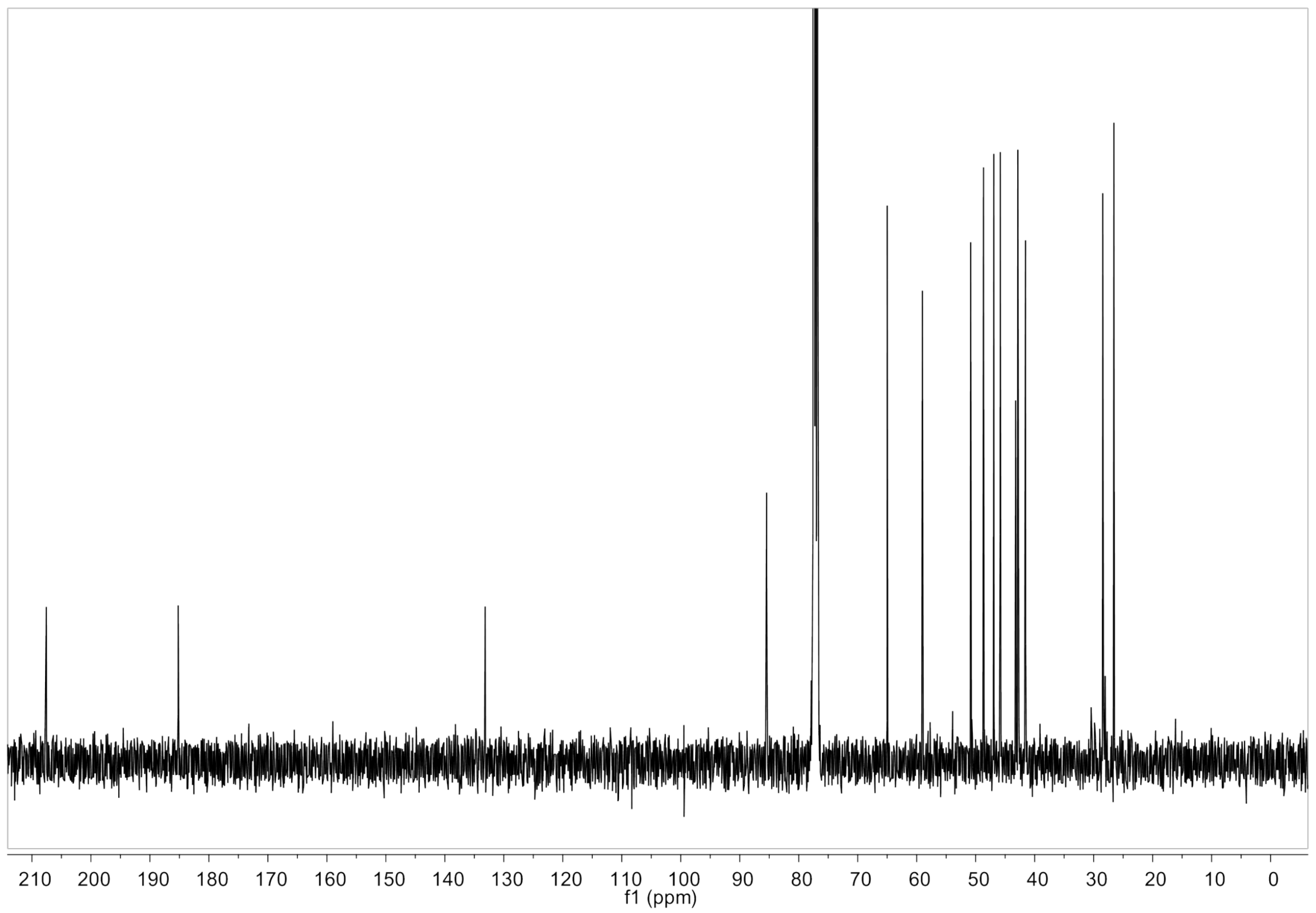

9 
${ }^{1} \mathrm{H}$ NMR (400 MHz, $\left.\mathrm{CDCl}_{3}\right)$ of xeromphalinone $\mathrm{E}(5)$

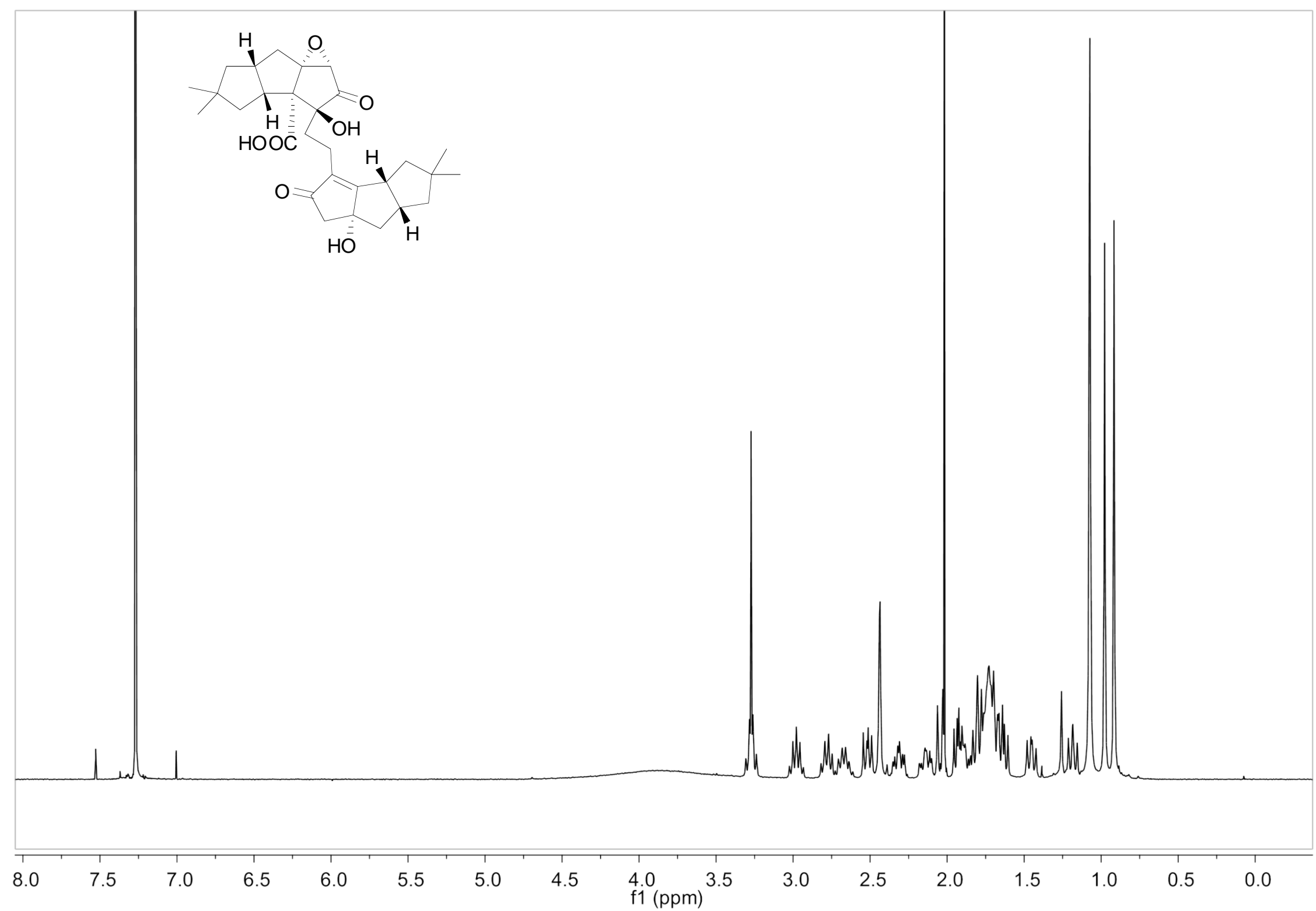


${ }^{13} \mathrm{C}$ NMR (101 MHz, $\mathrm{CDCl}_{3}$ ) of xeromphalinone $\mathrm{E}(5)$

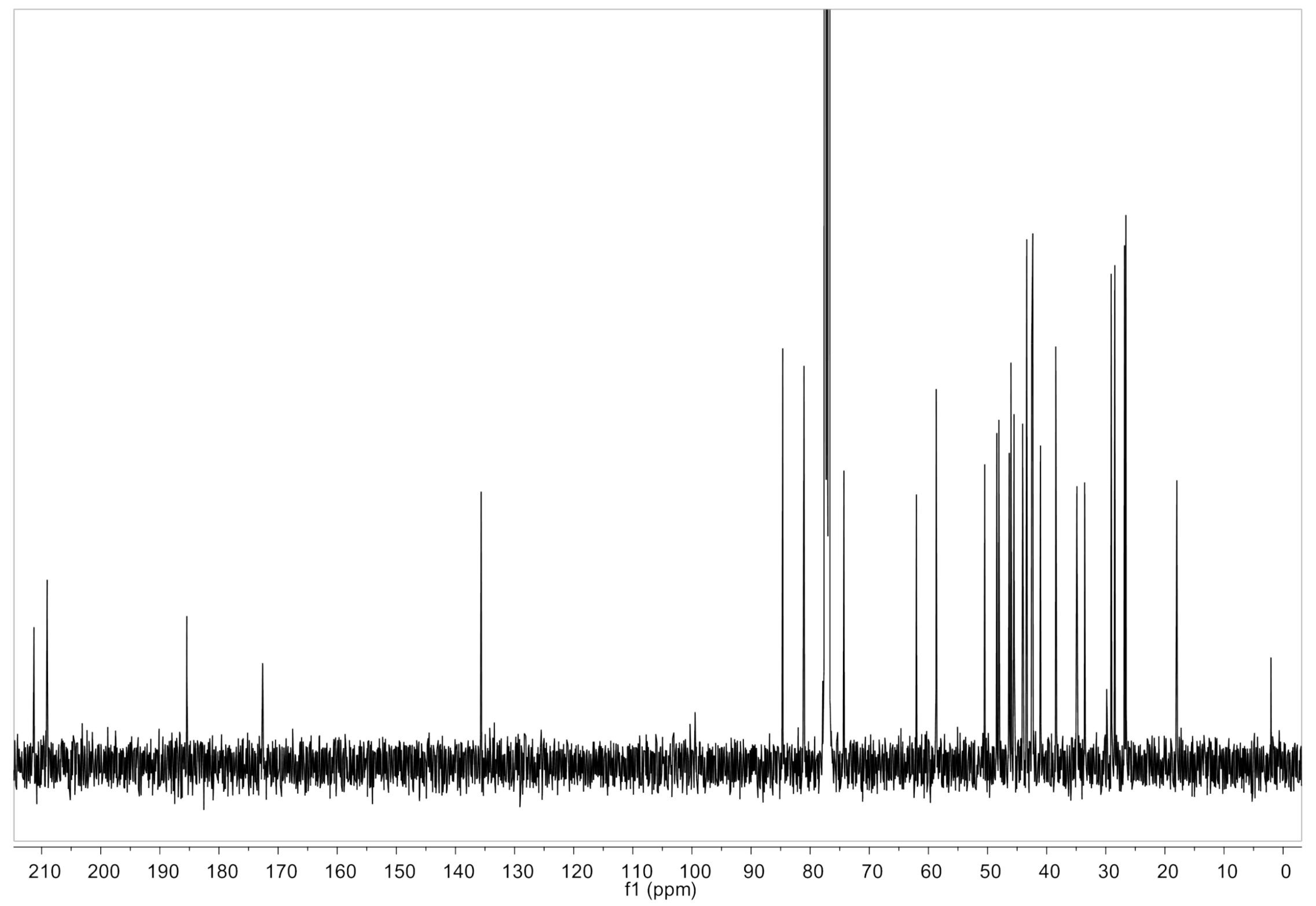


${ }^{1} \mathrm{H}$ NMR (400 MHz, $\left.\mathrm{CDCl}_{3}\right)$ of xeromphalinone $\mathrm{F}(6)$

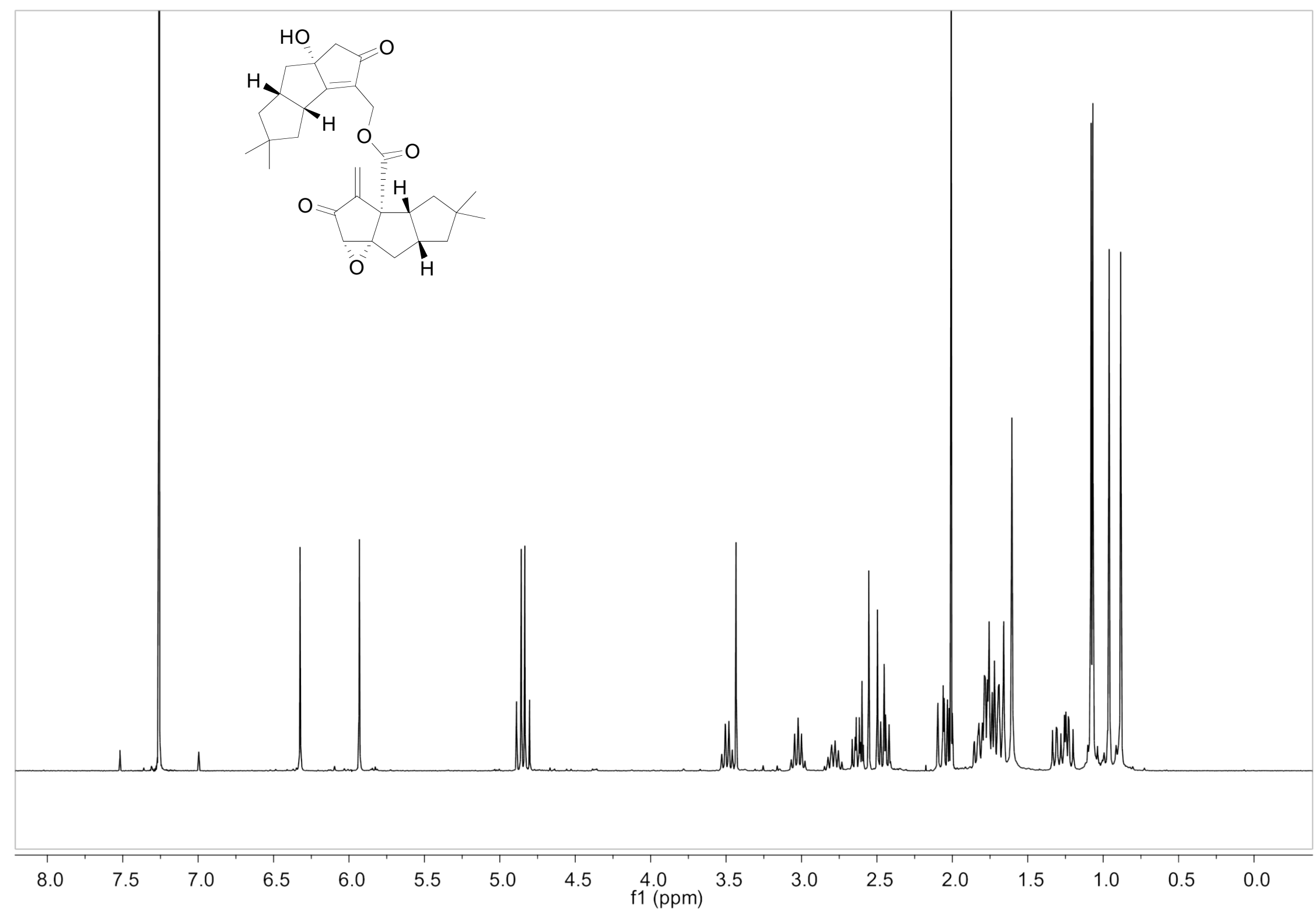


${ }^{13} \mathrm{C}$ NMR (101 MHz, $\mathrm{CDCl}_{3}$ ) of xeromphalinone $\mathrm{F}(6)$

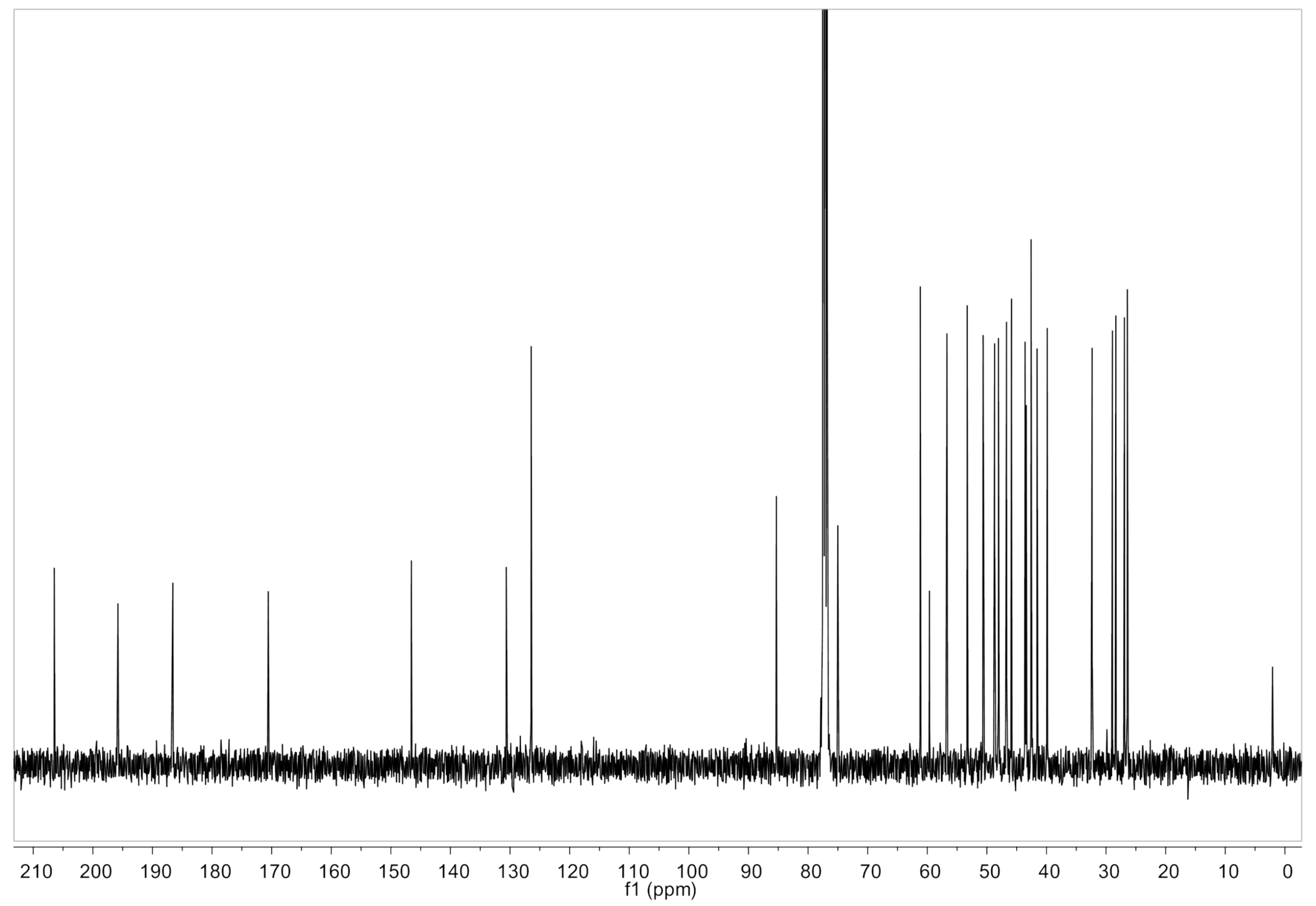


${ }^{1} \mathrm{H}$ NMR (400 MHz, $\mathrm{CDCl}_{3}$ ) of chlorostereone (7)

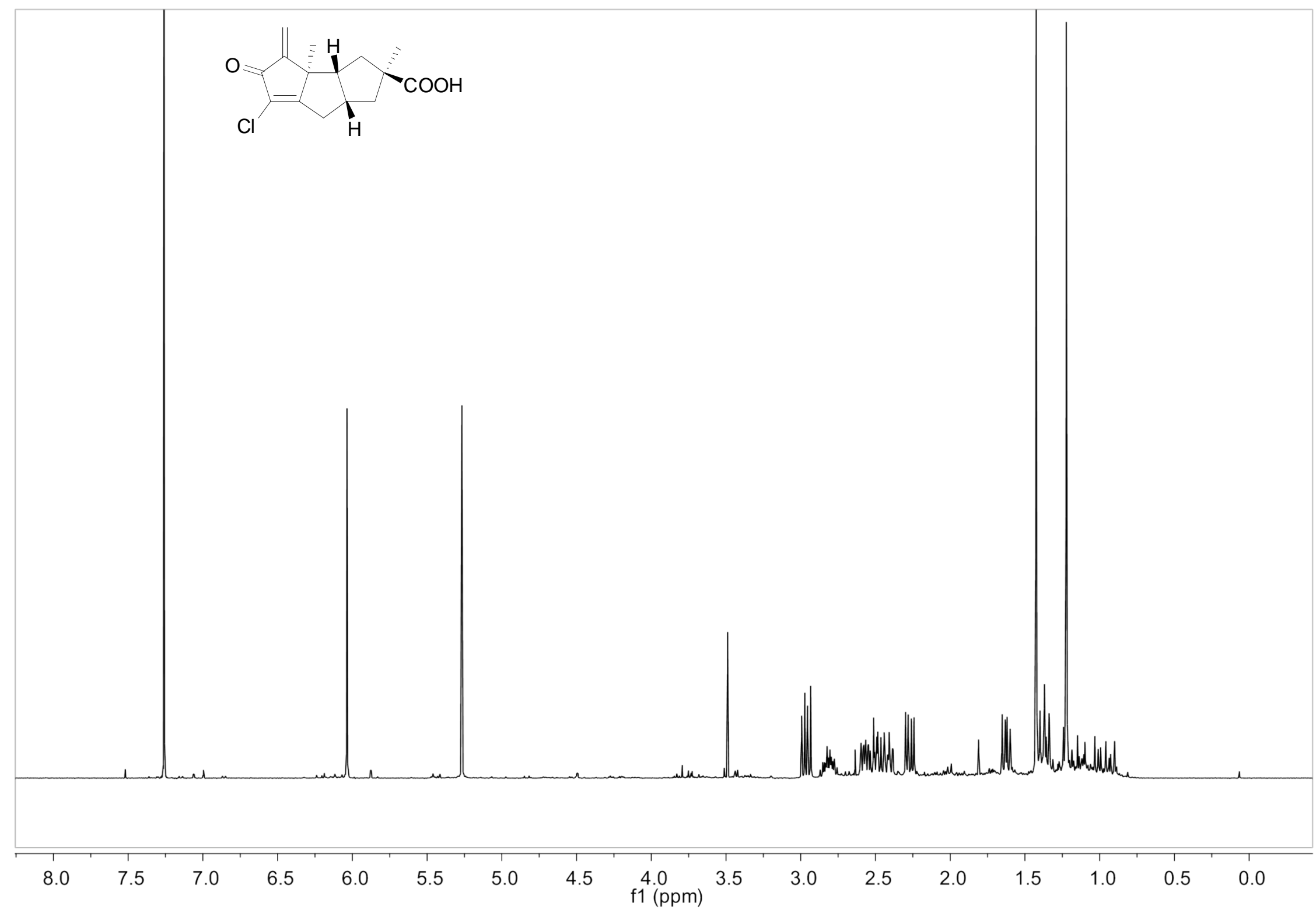


${ }^{13} \mathrm{C}$ NMR (101 MHz, $\mathrm{CDCl}_{3}$ ) of chlorostereone (7)

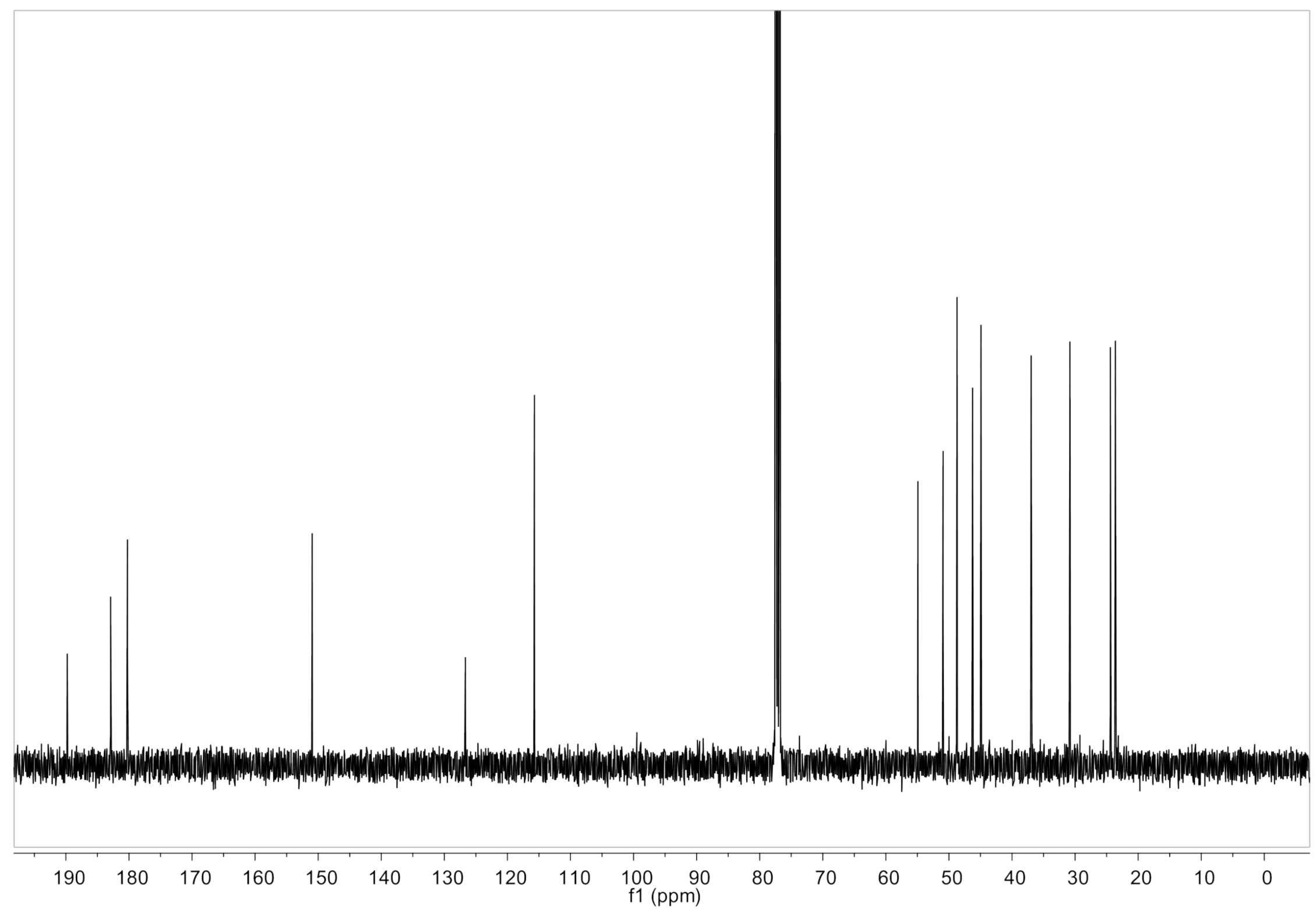


${ }^{1} \mathrm{H}$ NMR (400 MHz, $\left.\mathrm{CDCl}_{3}\right)$ of pleurocybellone $\mathrm{A}(8)$

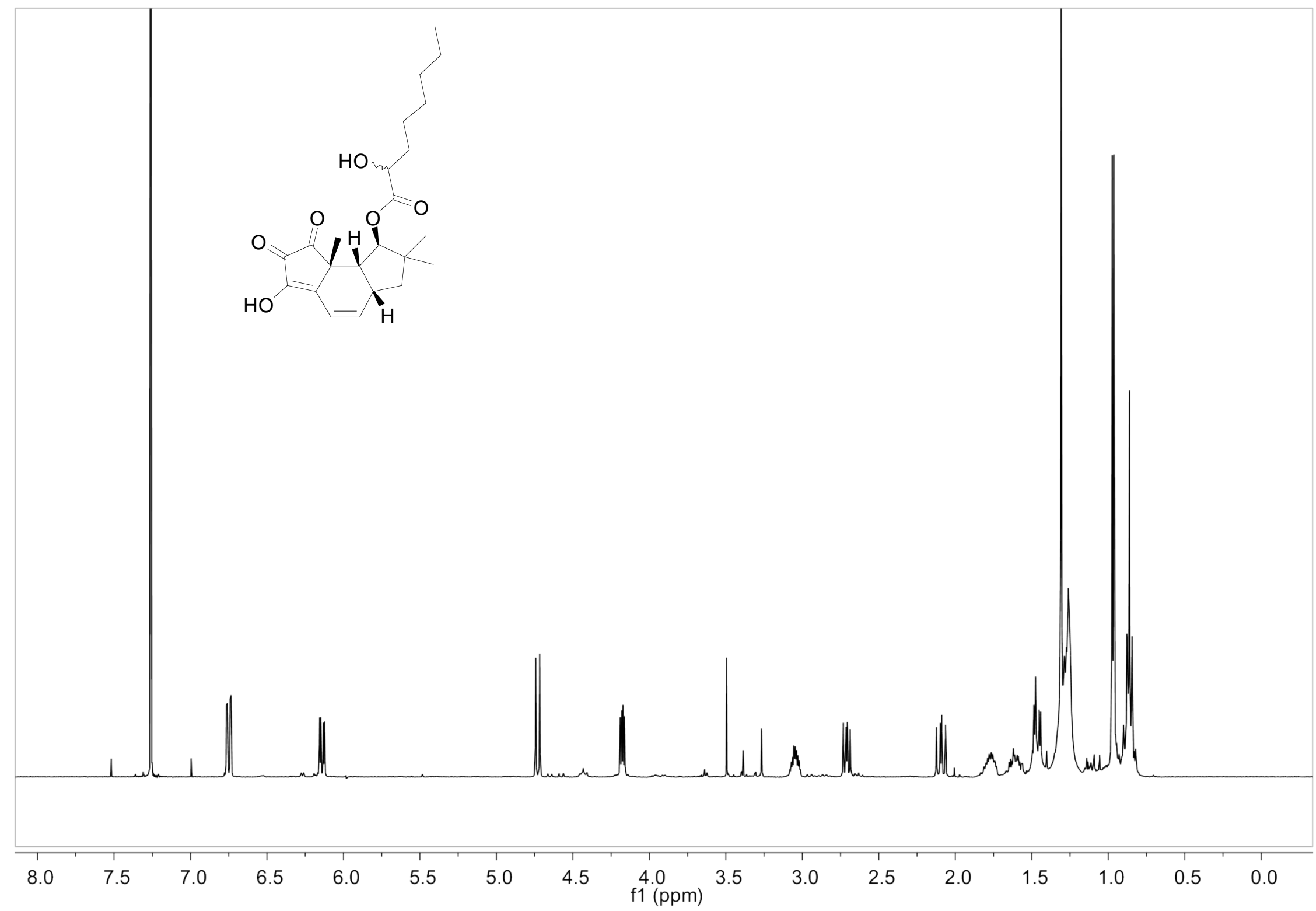


${ }^{13} \mathrm{C}$ NMR (101 MHz, $\mathrm{CDCl}_{3}$ ) of pleurocybellone A (8)

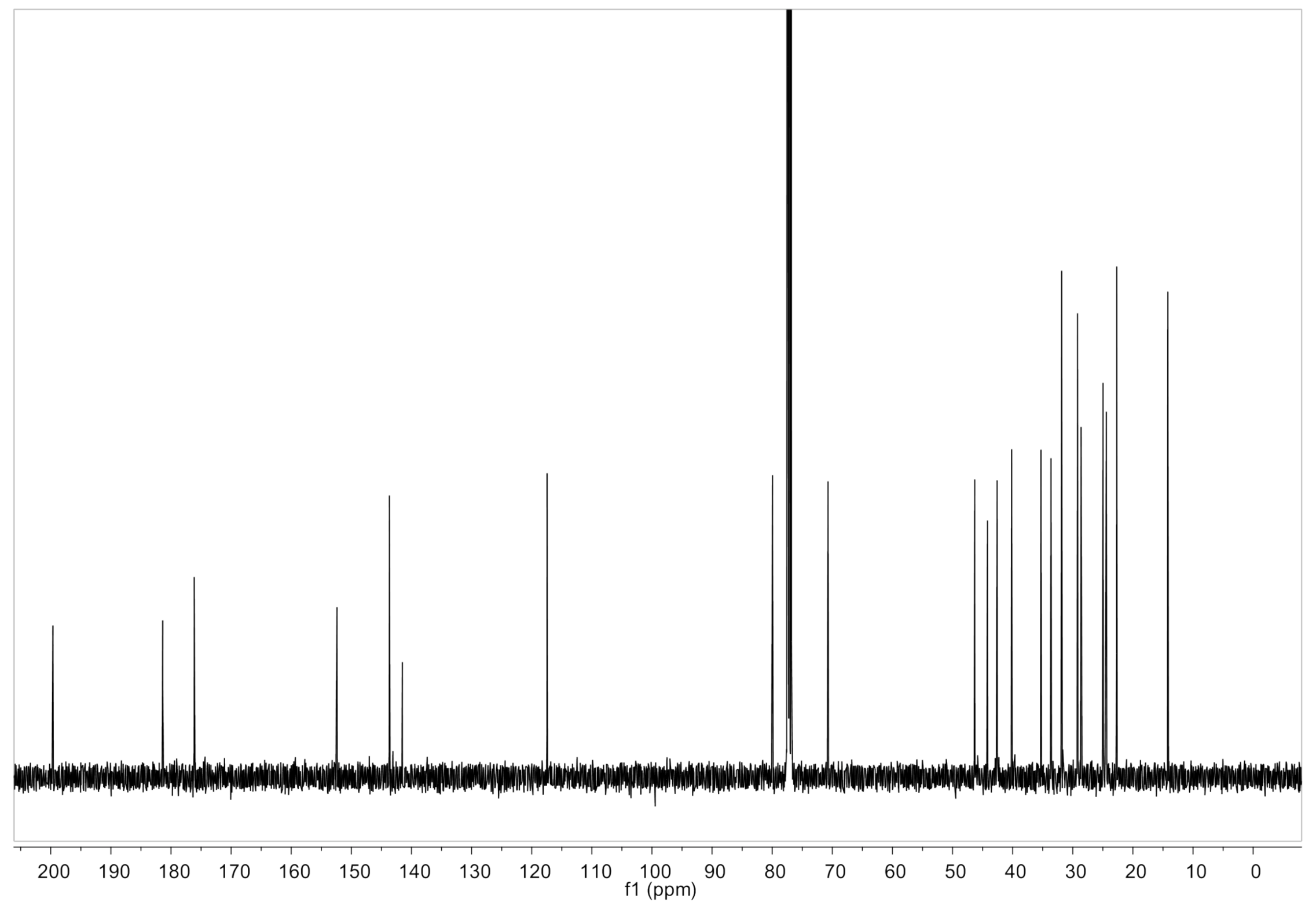

